\title{
External Cavity Laser With a Vertically Etched Silicon Blazed Grating
}

\author{
A. Lohmann and R. R. A. Syms, Member, IEEE
}

\begin{abstract}
An external cavity laser based on a stripe waveguide semiconductor optical amplifier, a ball lens, and a blazed diffraction grating fabricated by vertical deep reactive ion etching of silicon is demonstrated. Bandpass characteristics for a 12th-order grating are given. Tuning of an external cavity laser over a 120-nm spectral range is demonstrated, with a maximum single-mode fiber-coupled power of $1 \mathrm{~mW}$ and side-mode suppression ratio of $30 \mathrm{~dB}$.
\end{abstract}

Index Terms-External cavity, grating, microelectromechanical systems, semiconductor laser.

G REAT interest has been shown in frequency-tunable narrow line sources for optical communications [1]. One possible approach is to use a monolithic source such as a multisection distributed Bragg reflector laser. An alternative is offered by an external cavity source, which may allow a simpler and more stable tuning algorithm. Such sources are based on a movable reflective filter. Vertical-cavity surface-emitting lasers (VCSELs) equipped with multilayer dielectric mirrors have been developed by Bandwidth 9 Inc. [2] and Core Tek Inc. [3]. However, VCSELs suffer from the disadvantage of low output power compared with stripe-emitting devices, and require simultaneous development of the gain block and tuning mechanism.

External cavity lasers based on conventional stripe-waveguide semiconductor optical amplifiers (SOAs) and piezoelectrically actuated blazed gratings were originally developed in the late 1980s by BTRL [4] and CNET [5]. More recently, SOAs have been combined with mirrors [6], [7] and gratings [8] on micromachined electrostatic actuators by NTT and Iolon, respectively. The advantages of this approach are a reduction in size and cost, combined with an improvement in stability and reliability.

The most common geometries for an external cavity laser based on an SOA are the Littrow and Littman/Metcalf configurations, shown in Fig. 1(a) and (b), respectively. The former involves a single pass through a blazed grating, and the latter a double pass through a reflection grating via a further external mirror. In each case, rotation of a key component about a well-chosen virtual pivot point can avoid longitudinal mode hopping. Littrow cavities with conventionally fabricated blazed gratings were used by BTRL, and Littman cavities by Iolon.

\footnotetext{
Manuscript received May 2, 2002; revised September 10, 2002. This work was supported by Marconi plc and the Engineering and Physical Sciences Research Council.

The authors are with the Optical and Semiconductor Devices Section, Department of Electrical and Electronic Engineering, Imperial College, London SW7 2BT, U.K. (e-mail: r.syms@ic.ac.uk).

Digital Object Identifier 10.1109/LPT.2002.805762
}

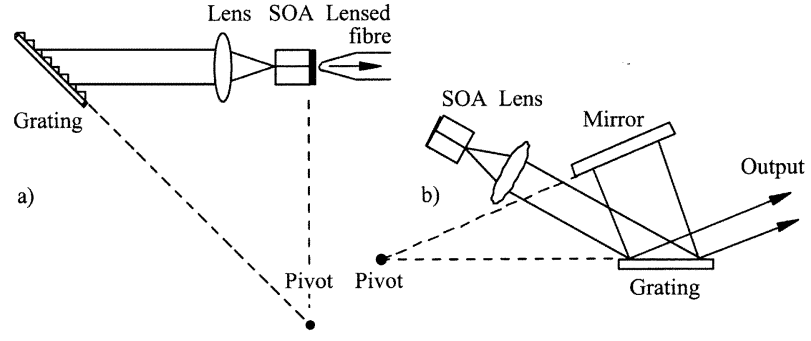

Fig. 1. Schematic of (a) Littrow and (b) Littman cavities.

In this letter, we demonstrate a laser based on a vertically etched silicon blazed grating arranged in a Littrow cavity. The fabrication approach used may allow eventual integration of the grating with a suitable positioning actuator.

In the Littrow configuration, retro-reflection occurs when $L \lambda / 2=\Lambda \sin (\theta)$, where $L$ is an integer order, $\lambda$ is the optical wavelength, $\Lambda$ is the grating period, and $\theta$ is the incidence angle. Gratings were designed for operation at $\lambda \approx 1.5 \mu \mathrm{m}$, with different values of $\Lambda(1.5 \sqrt{ } 2,3 \sqrt{ } 2,6 \sqrt{ } 2$, and $9 \sqrt{ } 2 \mu \mathrm{m}$, corresponding to $2 \mathrm{nd}, 4$ th, 8 th, and 12 th order at $\theta=45^{\circ}$ ). The fabrication process was very simple. A $3-\mu \mathrm{m}$-thick layer of resist was deposited, patterned into a staircase layout by contact lithography, and hard-baked to act as a surface mask. The silicon was then etched to $100 \mu \mathrm{m}$ depth in an inductively coupled plasma etcher using a cyclic etch-passivation process (see, e.g., [9]). After etching, residual resist was stripped in a plasma asher. Reflectivity was then enhanced using a sputtered layer of Au.

Fig. 2(a) shows a scanning electron microscope (SEM) photograph of a grating resolution test structure. Gratings of all the orders above were successfully fabricated. However, some initial rounding of the grating-tooth profile caused by the use of contact lithography was observed, together with a gradual degradation of the profile with depth due to mask erosion. The former became more significant as the grating order was reduced, and the latter as the grating depth increased. Greater fidelity in transferring the grating profile could clearly be obtained by (for example) replacing contact lithography with direct-write e-beam lithography, and by substituting the resist-hard mask with a silica mask patterned by reactive ion etching.

Fig. 2(b) shows an enlarged view of a 100- $\mu$ m-deep 12thorder grating, showing that the tooth profile is transferred with reasonable fidelity through the full etch depth. At higher magnifications, horizontal surface scallops caused by the cyclic nature of the etching process can be seen, together with rough vertical surface features. Both features will give rise to scatter. However, 


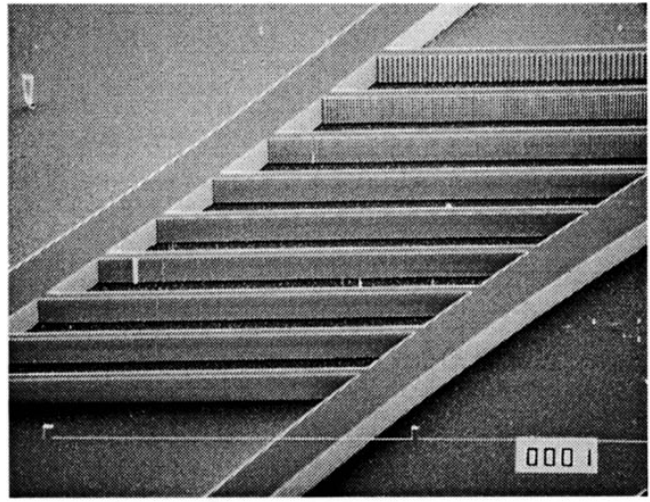

(a)

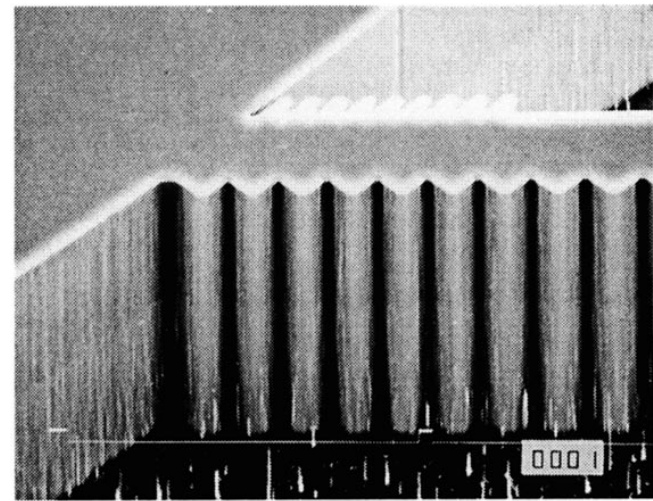

(b)

Fig. 2. Blazed gratings fabricated by deep reactive ion etching of Si. (a) Test pattern. (b) Enlarged view of 12th-order grating.

these gratings were found to have reasonable and repeatable optical performance, which was mainly set by size of the illuminating beam.

Grating performance was estimated by measuring the reflected power using an optical spectrum analyzer equipped with an internal 1550 -nm edge-emitting light-emitting diode (LED) source (Agilent 86142B), and comparing the result with the reflectivity of a plane Au-metallized Si surface. The incident and reflected beams were routed by a pigtailed $3-\mathrm{dB}$ fiber coupler and collimated by a $0.5-\mathrm{mm}$ diameter, 0.23 -pitch gradient index rod lens (GRINTECH GmbH, Germany) into a beam of approximately $100-\mu \mathrm{m}$ diameter.

Fig. 3 shows the spectral variation of reflectivity for a $100-\mu \mathrm{m}$-deep 12 th-order grating. The peak reflectivity is $5.5 \mathrm{~dB}$ lower than the plane mirror. This result suggests that some power is lost by scattering and by diversion into other diffraction orders. The equivalent result for a plane vertically etched surface is $2.5 \mathrm{~dB}$, suggesting a similar excess loss in the grating due to scattering, the remaining $3.0 \mathrm{~dB}$ being lost in other orders. The half-power bandwidth is approximately $20 \mathrm{~nm}$. Similar bandpass characteristics were obtained from a commercial-blazed grating (from Elliot Scientific, with blaze angle of $17^{\circ}$ and 1200 grooves $/ \mathrm{mm}$ ) when illuminated by a beam of the same diameter.

The gain block was a stripe guide InGaAsP device from OptoSpeed (LCSH1550-TAP with antireflection (AR)/high-reflection (HR) coatings). The emission was extracted using a lensed

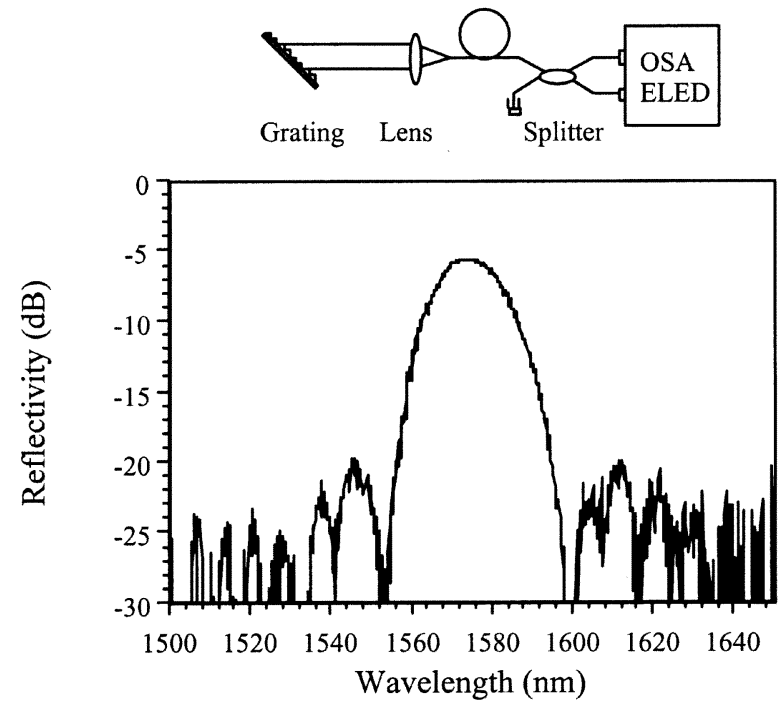

Fig. 3. Bandpass characteristic for a vertically etched 12th-order grating

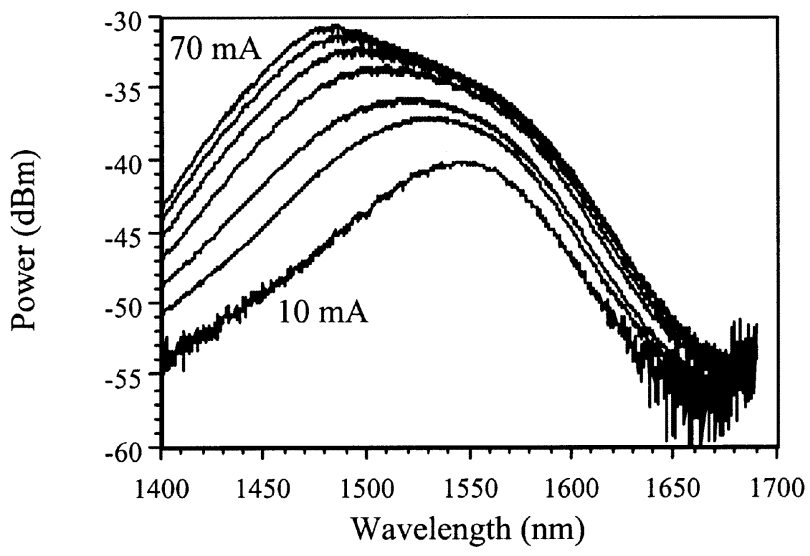

Fig. 4. Light-current characteristic for OptoSpeed LCSH-1550 TAP HR/AR gain block.

single-mode fiber with a tip radius of $9 \mu \mathrm{m}$, AR coated for 1550-nm wavelength (Seikho Gihken). Fig. 4 shows the spectral variation of the SOA emission, at currents ranging from 10 to $70 \mathrm{~mA}$ in steps of $10 \mathrm{~mA}$. The emission is smooth, with no visible internal resonances. The peak lies near $\lambda=1550 \mathrm{~nm}$ at low current, shifting to shorter wavelengths as the current rises.

External cavity lasers were constructed using this gain block, a 0.3-mm-diameter ball lens (Edmund Optics) and a $100-\mu \mathrm{m}$-deep 12th-order blazed grating. The components were aligned using separate micromanipulator stages, which resulted in some instability and poor cooling of the SOA. Cavities with lengths ranging from 25 to $4 \mathrm{~mm}$ were constructed.

The optical power output was measured by integrating over all lines using a calibrated meter (Newport 1830-C). Fig. 5 shows the light-current characteristic for a cavity length of $4 \mathrm{~mm}$, with the cavity tuned to $1550-\mathrm{nm}$ wavelength. The threshold current is $\approx 15 \mathrm{~mA}$, and the slope efficiency $d P / d I$ is $\approx 0.023 \mathrm{~mW} / \mathrm{mA}$ during lasing. The drive current was limited to avoid destroying the SOA by thermal runaway. Despite this, fiber-coupled powers of $>1.0 \mathrm{~mW}$ (single mode) and $>1.5 \mathrm{~mW}$ (multimode) have been obtained. 


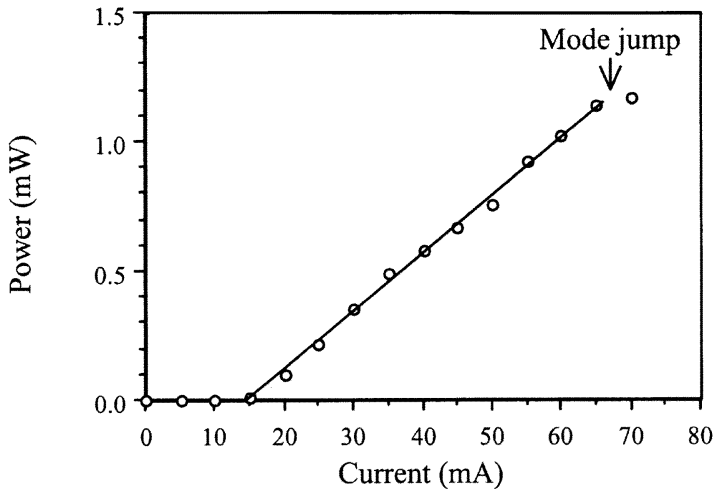

Fig. 5. Light-current characteristic for an external cavity laser based on a 12th-order grating.

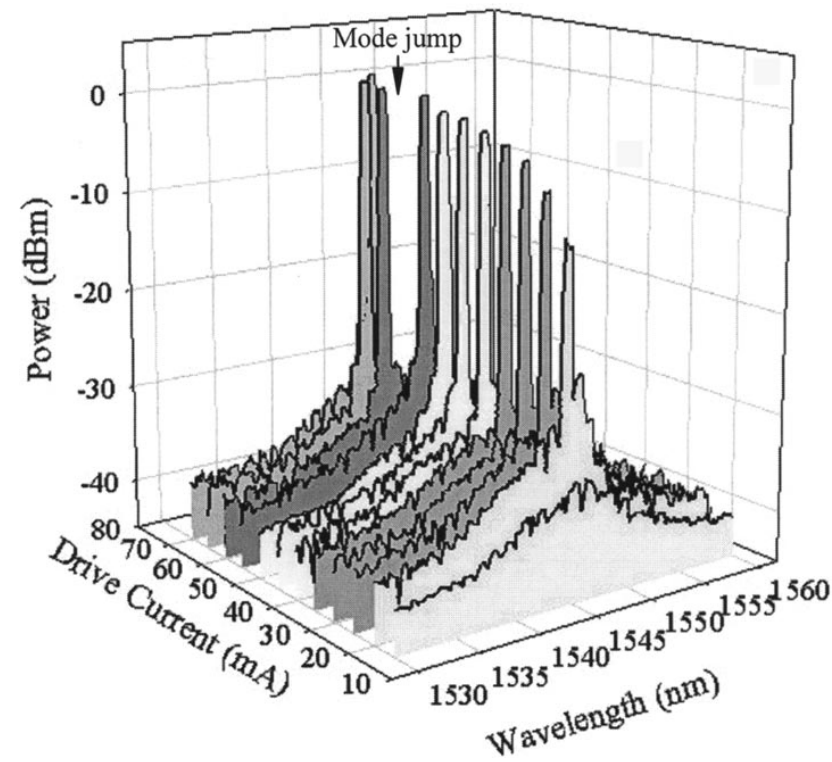

Fig. 6. Output spectra obtained at different currents, showing mode jump at high current.

As the current rises, the laser shows the instabilities typical of an external cavity device. For example, the kink at the extremity of the light-current characteristic of Fig. 5 is due to a mode jump. Fig. 6 shows the output spectra obtained at different drive currents. There is clearly a discrete shift in the position of the main lasing mode to a shorter wavelength at high current, associated with the gradual alteration of the emission spectrum of the SOA previously shown in Fig. 4. At higher currents still, the output is multimode.

The laser was tuned by mechanical rotation of the grating. Fig. 7 shows the spectral variation of emission at several angles of incidence, for a drive current of $60 \mathrm{~mA}$. Single-mode operation can clearly be maintained over a wide spectral range, from 1450 to $1570 \mathrm{~nm}$. This range is limited by the emergence of additional modes arising from higher diffraction orders. The side-mode suppression ratio (SMSR) is $\approx 30 \mathrm{~dB}$ near $1530 \mathrm{~nm}$.

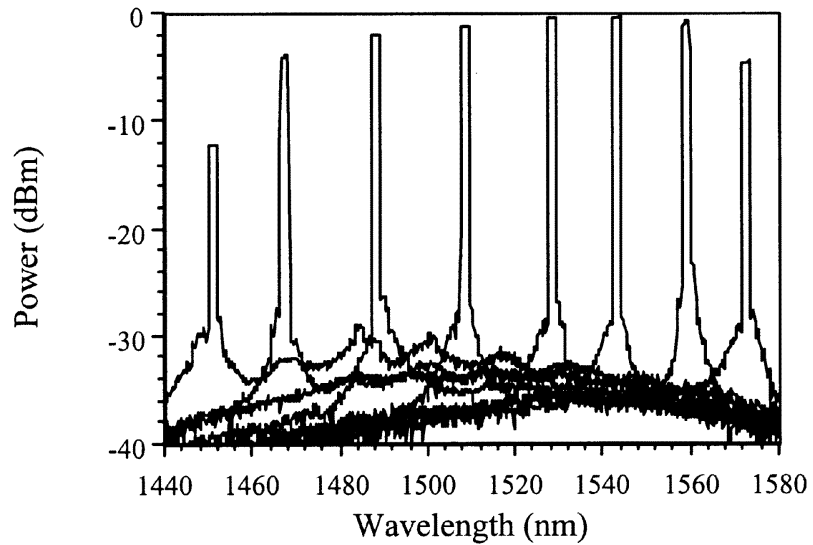

Fig. 7. Tuning characteristic for an external cavity laser based on a 12th-order grating.

The simple demonstration has shown that vertically etched diffractive-silicon optical components can have usable performance in an external cavity laser. Work is now underway to improve the optical quality and reduce the order of the grating, and to integrate the grating tuning mechanism (an elastic cantilever suspension and an electrostatic drive) by deep etching and undercut of bonded silicon-on-insulator material.

\section{ACKNOWLEDGMENT}

The authors are grateful to Analog Devices Belfast for performing the deep silicon etching.

\section{REFERENCES}

[1] B. Pezeshki, "New approaches to laser tuning," Opt. Photon. News, pp. 34-38, May 2001.

[2] M. Y. Li, G. S. Li, and C. J. Chang-Hasnain, "Top-emitting micromechanical VCSEL with a $31.6 \mathrm{~nm}$ tuning range," IEEE Photon. Technol. Lett., vol. 10, pp. 18-20, Jan. 1998.

[3] D. Vakhshoori, P. Tayebati, C.-C. Lu, M. Azimi, P. Wang, J.-H. Zhou, and E. Canoglu, " $2 \mathrm{~mW} \mathrm{CW}$ singlemode operation of a tunable $1550 \mathrm{~nm}$ vertical cavity surface emitting laser with $50 \mathrm{~nm}$ tuning range," Electron. Lett., vol. 35, pp. 900-901, 1999.

[4] J. Mellis, S. A. Al-Chalabi, K. H. Cameron, R. Wyatt, J. C. Regnault, W. J. Devlin, and M. C. Brain, "Miniature packaged external-cavity semiconductor laser with $50 \mathrm{GHz}$ continuous electrical tuning range," Electron. Lett., vol. 24, pp. 988-989, 1988.

[5] F. Favre and D. Le Guen, " $82 \mathrm{~nm}$ of continuous tunability for an external cavity semiconductor laser," Electron. Lett., vol. 27, pp. 183-184, 1991.

[6] Y. Uenishi, M. Tsugai, and M. Mehregany, "Hybrid-integrated laser diode micro-external mirror fabricated by (110) silicon micromachining," Electron. Lett., vol. 31, pp. 965-966, 1995.

[7] Y. Uenishi, K. Honma, and S. Nagaoka, "Tunable laser diode using a nickel micromachined external mirror," Electron. Lett., vol. 32, pp. 1207-1208, 1996.

[8] J. D. Berger, Y. Zhang, J. D. Grade, H. Lee, S. Hrinya, and J. H. Jerman, "Widely tunable external cavity diode laser based on a MEMS electrostatic rotary actuator," presented at the OFC 2001, Anaheim, CA, Mar. 17-22, 2001, Paper Tuj2.

[9] A. M. Hynes, H. Ashraf, J. K. Bhardwaj, J. Hopkins, I. Johnston, and J. N. Shepherd, "Recent advances in silicon etching for MEMS using the ASETM process," Sens. Actuators, vol. 74, pp. 13-17, 1999. 